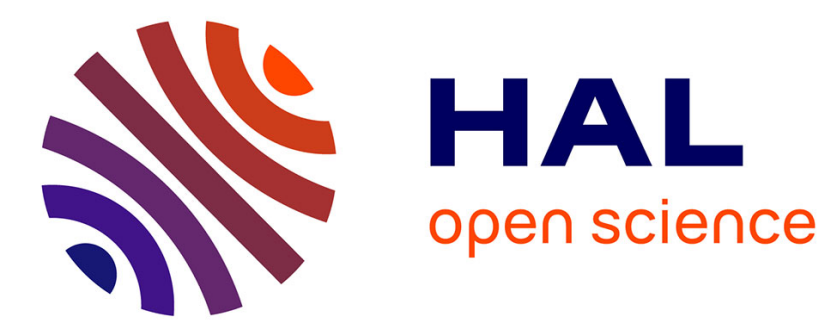

\title{
Delay-robust stabilization of a hyperbolic PDE-ODE system
}

\author{
Jean Auriol, Federico Bribiesca Argomedo, David Bou Saba, Michaël Di \\ Loreto, Florent Di Meglio
}

\section{- To cite this version:}

Jean Auriol, Federico Bribiesca Argomedo, David Bou Saba, Michaël Di Loreto, Florent Di Meglio. Delay-robust stabilization of a hyperbolic PDE-ODE system. Automatica, 2018, 95, pp.494 - 502. 10.1016/j.automatica.2018.06.033 . hal-01823656

\section{HAL Id: hal-01823656 https://hal.science/hal-01823656}

Submitted on 2 Apr 2019

HAL is a multi-disciplinary open access archive for the deposit and dissemination of scientific research documents, whether they are published or not. The documents may come from teaching and research institutions in France or abroad, or from public or private research centers.
L'archive ouverte pluridisciplinaire HAL, est destinée au dépôt et à la diffusion de documents scientifiques de niveau recherche, publiés ou non, émanant des établissements d'enseignement et de recherche français ou étrangers, des laboratoires publics ou privés. 


\title{
Delay-robust stabilization of a hyperbolic PDE-ODE system
}

\author{
Jean Auriol ${ }^{\text {a }}$, Federico Bribiesca-Argomedo ${ }^{\mathrm{b}}$, David Bou Saba ${ }^{\mathrm{b}}$, \\ Michael Di Loreto ${ }^{\mathrm{b}}$, Florent Di Meglio ${ }^{\mathrm{a}}$ \\ ${ }^{a}$ MINES ParisTech, PSL Research University, CAS - Centre Automatique et Systèmes, 60 bd St Michel, 75006 Paris, France \\ ${ }^{\mathrm{b}}$ Univ Lyon, INSA Lyon, Laboratoire Ampère CNRS UMR5005, F-69621, Villeurbanne, France
}

\begin{abstract}
We detail in this article the development of a delay-robust stabilizing feedback control law for a linear ordinary differential equation coupled with two linear first order hyperbolic equations in the actuation path. The proposed method combines the use of a backstepping approach, required to construct a cancelling feedback for the in-domain coupling terms of the PDEs, with a second change of variables that reduces the stabilization problem of the PDE-ODE system to that of a time-delay system for which a predictor can be constructed. The proposed controller can be tuned, with some restrictions imposed by the system structure, either by adjusting a reflection coefficient left on the PDE after the backstepping transformation, or by choosing the pole placement on the ODE when constructing the predictor, enabling a trade-off between convergence rate and delay-robustness. The proposed feedback law is finally proved to be robust to small delays in the actuation
\end{abstract}

Key words: Hyperbolic Partial Differential Equations; Time-Delay Systems ; Stabilization; Backstepping.

\section{Introduction}

In this paper we develop a linear feedback control law that achieves delay-robust stabilization of a system of two heterodirectional linear first-order hyperbolic Partial Differential Equations (PDEs) coupled through the boundary to an Ordinary Differential Equation (ODE). The proposed design works for all systems within the considered class for which delay-robust stabilization by such a feedback operator can be expected, see [17]. We achieve this by partially leveraging the backstepping design in [11] and complementing it with a predictor-based controller after an adequate reformulation using a timedelay approach.

The control of systems of coupled ODEs and hyperbolic PDEs is a very active research topic, see for instance $[5,7,11,22]$. It naturally arises when considering delays (that can be seen as first-order hyperbolic PDEs) in the actuating and sensing paths of ODEs $[5,6,12,24,27]$. A recurrent practical motivation for the study of such systems is the attenuation of mechanical vibrations in drilling applications, where the

Email addresses: jean.auriol@mines-paristech.fr (Jean Auriol), federico.bribiesca@insa-lyon.fr (Federico Bribiesca-Argomedo), david.bou-saba@insa-lyon.fr (David Bou Saba), michael.di-loreto@insa-lyon.fr (Michael Di Loreto), florent.di_meglio@mines-paristech.fr (Florent Di Meglio). hyperbolic PDEs represent axial and torsional stress propagation (waves) along the drill string, while the ODE models the Bottom Hole Assembly (BHA) dynamics. An thorough review of drilling vibrations models is available in [23]

The backstepping approach was first used in [16] to deal with hyperbolic PDE-ODE couplings where actuator and sensor delays are explicitly compensated. While this problem had already been tackled by the Smith predictor [25], the reformulation of the delay as a linear ODE enabled numerous related problems to be tackled, most notably the presence of non-constant and uncertain delays $[4,6]$. Recently, the general problem of stabilizing an ODE with a system of first-order linear hyperbolic PDEs in the actuator path was solved in [11] using a backstepping transformation that maps the fully interconnected system into a cascade of exponentially stable subsystems. This was achieved by canceling, among other terms, the reflection at the controlled boundary. While this approach enables the design of predictor-like feedback laws, and is mathematically correct, it does not take into account the impact on stability of small delays in the feedback loop (delay-robustness).

It has been observed, see for instance [10,17], that for many feedback systems, the introduction of arbitrarily small time-delays in the loop may cause instability under linear state feedback. In particular, for coupled linear hyperbolic systems, recent contributions [1] have highlighted the necessity of a change of paradigm in order to achieve delay-robust stabilization. It turns out that, 
in some cases, one must preserve some reflection at the boundaries in order to maintain the delay robustness of the control. Also, in [1], the authors use the backstepping transformation in order to rewrite the system as a neutral equation with distributed delays. This opens the perspective of adapting stability analysis methods for time delay systems, such as those developed in $[9,15,20,21]$ on hyperbolic PDE systems.

The main contribution of this paper is to provide a new design for a state-feedback law for a PDE-ODE system that ensures the delay-robust stabilization. Delayrobustness is ensured by preserving some proximal reflection terms in the control law. This is done by means of an additional degree of freedom enabling a trade-off between convergence rate in the absence of delay and delay-robustness. Note that the approach of [1] cannot be directly extended since the system naturally features several feedback loops or couplings that can be sources of instabilities.

Our approach is the following: (i) A backstepping transformation (and associated feedback operator) is constructed, removing the in-domain couplings present in the PDEs and possibly attenuating the reflections on the controlled side (depending on the choice of a tuning parameter). Without these in-domain couplings, the new system can be rewritten as a neutral delay differential equation. (ii) Using the structure of the obtained equation, we construct a non-invertible operator that preserves detectability in order to reduce the stabilization problem of the neutral system to that of a linear ODE system with delayed input, for which a statepredictor feedback law is constructed. (iii) Finally, the delay-robustness properties of the system are studied by means of an algebraic analysis in the Laplace domain.

The paper is organized as follows. In Section 2 we introduce the model equations and the notations. In Section 3 , we present the stabilization result: using a backstepping transformation, we first dissociate the ODE and the PDE. The original system can then be rewritten as a distributed delay equation for which it is possible to derive a stabilizing control law. The corresponding feedback system is proved to be stable to small delays in Section 4. Finally, some simulation results are given in Section 5.

\section{Problem Formulation}

\subsection{Definitions and notations}

In this section we detail the notations used through this paper. For any integer $p>0,\|\cdot\|_{\mathbb{R}^{p}}$ is the classical euclidean norm on $\mathbb{R}^{p}$. We denote by $L^{1}([0,1], \mathbb{R})$, or $L^{1}([0,1])$ if no confusion arises, the space of real-valued functions defined on $[0,1]$ whose absolute value is integrable. This space is equipped with the standard $L^{1}$ norm, that is, for any $f \in L^{1}([0,1])$

$$
\|f\|_{L^{1}}=\int_{0}^{1}|f(x)| \mathrm{d} x .
$$

We denote $L^{2}([0,1], \mathbb{R})$ the space of real-valued squareintegrable functions defined on $[0,1]$ with the standard $L^{2}$ norm, i.e., for any $f \in L^{2}([0,1], \mathbb{R})$

$$
\|f\|_{L^{2}}^{2}=\int_{0}^{1} f^{2}(x) \mathrm{d} x .
$$

The set $L^{\infty}([0,1], \mathbb{R})$ denotes the space of bounded realvalued functions defined on $[0,1]$ with the standard $L^{\infty}$ norm, i.e., for any $f \in L^{\infty}([0,1], \mathbb{R})$

$$
\|f\|_{L^{\infty}}=\sup _{x \in[0,1]}|f(x)| .
$$

In the following, for $(u, v, X) \in\left(L^{2}([0,1])\right)^{2} \times \mathbb{R}^{p}$, we define the norm

$$
\|(u, v, X)\|=\|u\|_{L^{2}}+\|v\|_{L^{2}}+\|X\|_{\mathbb{R}^{p}}
$$

The $\operatorname{set} \mathcal{C}^{p}([0,1])$ (with $p \in \mathbb{N} \cup\{\infty\}$ ) stands for the space of real-valued functions defined on $[0,1]$ that are $p$ times differentiable and whose $p$-th derivative is continuous. The set $\mathcal{T}$ is defined as

$$
\mathcal{T}=\left\{(x, \xi) \in[0,1]^{2} \text { s.t. } \xi \leq x\right\} .
$$

$\mathcal{C}(\mathcal{T})$ stands for the space of real-valued continuous functions on $\mathcal{T}$. For a positive real $k$ and two reals $a<b$, a function $f$ defined on $[a, b]$ is said to be $k$-Lipschitz if for all $(x, y) \in[a, b]^{2}$, it satisfies $|f(x)-f(y)| \leq k|x-y|$. The symbol $I_{p}$ (or $I$ if no confusion arises) represents the $p \times p$ identity matrix. We use the notation $\hat{f}(s)$ for the Laplace transform of a function $f(t)$, provided it is well defined. The set $\mathcal{A}$ stands for the convolution Banach algebra of BIBO-stable generalized functions in the sense of [26]. A function $g(\cdot)$ belongs to $\mathcal{A}$ if it can be expressed as

$$
g(t)=g_{r}(t)+\sum_{i=0}^{\infty} g_{i} \delta\left(t-t_{i}\right)
$$

where $g_{r} \in L^{1}\left(\mathbb{R}^{+}, \mathbb{R}\right), \sum_{i \geq 0}\left|g_{i}\right|<\infty, 0=t_{0}<t_{1}<\ldots$ and $\delta(\cdot)$ is the Dirac distribution. The associated norm is

$$
\|g\|_{\mathcal{A}}=\left\|g_{r}\right\|_{L^{1}}+\sum_{i \geq 0}\left|g_{i}\right| .
$$

The set $\hat{\mathcal{A}}$ of Laplace transforms of elements in $\mathcal{A}$ is also a Banach algebra with associated norm

$$
\|\hat{g}\|_{\hat{\mathcal{A}}}=\|g\|_{\mathcal{A}} \text {. }
$$

\subsection{System under consideration}

We consider a class of systems consisting of an ODE coupled to two heterodirectional first-order linear hyperbolic systems in the actuation path, depicted schematically in Figure 1. More precisely, we consider systems of 


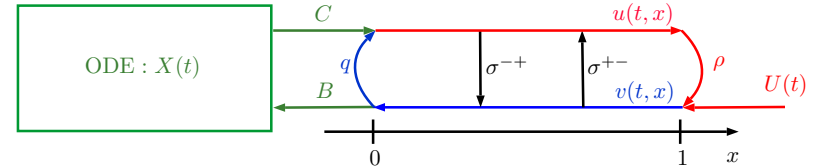

Fig. 1. Schematic representation of the system (3)-(6)

the form:

$$
\begin{aligned}
u_{t}(t, x)+\lambda u_{x}(t, x) & =\sigma^{+-}(x) v(t, x) \\
v_{t}(t, x)-\mu v_{x}(t, x) & =\sigma^{-+}(x) u(t, x) \\
\dot{X}(t) & =A X(t)+B v(t, 0),
\end{aligned}
$$

evolving in $\{(t, x)$ s.t. $t>0, x \in[0,1]\}$, with the boundary conditions

$$
\begin{aligned}
& u(t, 0)=q v(t, 0)+C X(t) \\
& v(t, 1)=\rho u(t, 1)+U(t),
\end{aligned}
$$

where $X \in \mathbb{R}^{p}$ is the ODE state, $u(t, x) \in \mathbb{R}$ and $v(t, x) \in$ $\mathbb{R}$ are the PDE states and $U(t)$ is the control input. The in-domain coupling terms $\sigma^{-+}$and $\sigma^{+-}$belong to $\mathcal{C}^{0}([0,1])$, the boundary coupling terms $q \neq 0$ (distal reflexion) and $\rho$ (proximal reflexion), and the velocities $\lambda$ and $\mu$ are constants. Furthermore, the velocities verify

$$
-\mu<0<\lambda \text {. }
$$

The initial conditions of the state $(u, v)$ are denoted $u_{0}$ and $v_{0}$ and are assumed to belong to $L^{2}([0,1], \mathbb{R})$ and we consider only weak $L^{2}$ solutions to the system. The initial condition of the ODE (5) is denoted $X_{0}$. The resulting system (3)-(6) is well-posed [3, Theorem A.6, page 254].

Remark that this system naturally features several couplings that can be source of instabilities. Also, the results of this paper can be extended to the case $q=0$ with a slight modification of the backstepping transformation.

\subsection{Control problem}

The goal of this paper is to design a feedback control law $U=\mathcal{K}[(u, v, X)]$ where $\mathcal{K}:\left(L^{2}[0,1]\right)^{2} \times \mathbb{R}^{p} \rightarrow \mathbb{R}$ is a linear operator, such that:

- the state $(u, v, X)$ of the resulting feedback system (3)-(6) exponentially converges to its zero equilibrium (stabilization problem), i.e. there exist $\kappa_{0} \geq 0$ and $\nu>0$ such that for any initial condition $\left(u_{0}, v_{0}, X_{0}\right) \in\left(L^{2}[0,1]\right)^{2} \times \mathbb{R}^{p}$

$$
\|(u, v, X)\| \leq \kappa_{0} \mathrm{e}^{-\nu t}\left\|\left(u_{0}, v_{0}, X_{0}\right)\right\|, t \geq 0 .
$$

- the resulting feedback system (3)-(6) is robustly stable with respect to small delays in the loop (delayrobustness), i.e. there exists $\delta^{\star}>0$ such that for any $\delta \in\left[0, \delta^{\star}\right]$, the control law $U(t-\delta)$ still stabilizes $(3)-(6)$.
A control law that satisfies these two constraints is said to delay-robustly stabilize (in the sense of [17]) system (3)-(6).

In this paper, we make the two following assumptions:

Assumption 1 The pair $(A, B)$ is stabilizable, i.e. there exists a matrix $K$ such that $A+B K$ is Hurwitz.

Assumption 2 The proximal reflection $\rho$ and the distal reflection $q$ satisfy $|\rho q|<1$.

The first assumption (stabilizability of the ODE subsystem) is necessary for the stabilizability of the whole system, while the second assumption is required for the existence of a delay-robust linear feedback control. This second assumption is not restrictive since, if is not fulfilled, one could prove using arguments similar to those in [1] that the open-loop transfer function has an infinite number of poles in the complex closed right half-plane. Consequently (see [17, Theorem 1.2]), one cannot find any linear state feedback law $U(\cdot)$ that delay-robustly stabilizes (3)-(6).

\section{Design of the control law}

In this section we derive a control law that guarantees the stabilization of (3)-(6), following the methodology introduced above. Using a backstepping transformation, we map the original system to a simpler target system without the in-domain couplings. This new target system is then rewritten as a neutral delay differential equation. Finally, the stability of this equation is reduced to that of an ODE with input delay for which a stabilizing control is constructed. This control law will be shown to be robust to small delays in the next section.

\subsection{Backstepping transformation}

We derive a Volterra transformation to rewrite system (3)-(6) as a system of transport equations coupled with an ODE. In other words, the purpose of this transformation is to remove the in-domain coupling terms, while conserving (only attenuating) boundary couplings. Let us consider the linear map that associates to any element $(\alpha, \beta, X) \in\left(L^{2}([0,1])^{2} \times \mathbb{R}^{p}\right.$ the corresponding element $(u, v, X) \in\left(L^{2}([0,1])^{2} \times \mathbb{R}^{p}\right.$ as follows

$$
\begin{aligned}
u(t, x)= & \alpha(t, x)+\int_{0}^{x} L^{\alpha \alpha}(x, \xi) \alpha(t, \xi) \mathrm{d} \xi \\
& +\int_{0}^{x} L^{\alpha \beta}(x, \xi) \beta(t, \xi) \mathrm{d} \xi+\gamma_{0}(x) X(t), \\
v(t, x)= & \beta(t, x)+\int_{0}^{x} L^{\beta \alpha}(x, \xi) \alpha(t, \xi) \mathrm{d} \xi \\
& +\int_{0}^{x} L^{\beta \beta}(x, \xi) \beta(t, \xi) \mathrm{d} \xi+\gamma_{1}(x) X(t), \\
X(t)= & X(t) .
\end{aligned}
$$


This mapping is a Volterra integral transformation and is consequently invertible. The kernels $L^{\alpha \alpha}, L^{\alpha \beta}, L^{\beta \alpha}$ and $L^{\beta \beta}$ are defined on $\mathcal{T}$ introduced in $(2), \gamma_{0}$ and $\gamma_{1}$ are row vectors with $p$ components defined on $([0,1])$. They satisfy the following set of PDEs

$$
\begin{aligned}
& \lambda L_{x}^{\alpha \alpha}(x, \xi)+\lambda L_{\xi}^{\alpha \alpha}(x, \xi)=\sigma^{+-}(x) L^{\beta \alpha}(x, \xi) \\
& \lambda L_{x}^{\alpha \beta}(x, \xi)-\mu L_{\xi}^{\alpha \beta}(x, \xi)=\sigma^{+-}(x) L^{\beta \beta}(x, \xi) \\
& \mu L_{x}^{\beta \alpha}(x, \xi)-\lambda L_{\xi}^{\beta \alpha}(x, \xi)=-\sigma^{-+}(x) L^{\alpha \alpha}(x, \xi) \\
& \mu L_{x}^{\beta \beta}(x, \xi)+\mu L_{\xi}^{\beta \beta}(x, \xi)=-\sigma^{-+}(x) L^{\alpha \beta}(x, \xi)
\end{aligned}
$$

and ODEs

$$
\begin{aligned}
& \lambda \gamma_{0}^{\prime}(x)=-\gamma_{0}(x) A+\sigma^{+-}(x) \gamma_{1}(x)-\lambda L^{\alpha \alpha}(x, 0) C \\
& \mu \gamma_{1}^{\prime}(x)=\gamma_{1}(x) A-\sigma^{-+}(x) \gamma_{0}(x)+\lambda L^{\beta \alpha}(x, 0) C
\end{aligned}
$$

with the boundary conditions

$$
\begin{aligned}
& L^{\beta \alpha}(x, x)=-\frac{\sigma^{-+}(x)}{\lambda+\mu}, L^{\alpha \beta}(x, x)=\frac{\sigma^{+-}(x)}{\lambda+\mu} \\
& L^{\alpha \alpha}(x, 0)=\frac{\mu}{\lambda q} L^{\alpha \beta}(x, 0)-\frac{1}{\lambda q} \gamma_{0}(x) B \\
& L^{\beta \beta}(x, 0)=\frac{\lambda q}{\mu} L^{\beta \alpha}(x, 0)+\frac{1}{\mu} \gamma_{1}(x) B \\
& \gamma_{1}(0)=0, \quad \gamma_{0}(0)=0
\end{aligned}
$$

Note that equation (18) is only defined for $q \neq 0$. If $q=0$, the kernel equations have to be slightly adjusted (see [8] for instance) and the resulting target system would be slightly different. However the method presented in this paper can still be used.

Lemma 1 Consider system (11)-(20). There exists a unique solution $L^{\alpha \alpha}, L^{\alpha \beta}, L^{\beta \alpha}$ and $L^{\beta \beta}$ in $\mathcal{C}(\mathcal{T})$ and $\gamma_{0}, \gamma_{1}$ in $\left(\mathcal{C}^{1}([0,1])\right)^{p}$.

PROOF. This result follows, with some minor adaptations, from [11, Theorem 3.2]. The main idea consists on reinterpreting the ODEs in (15)-(16) as PDEs evolving in the triangular domain $\mathcal{T}$ with horizontal characteristic lines (since there is only an evolution along $x$ ) and then solving all the PDEs together. In this case, we extend the ODEs for $\gamma_{0}$ and $\gamma_{1}$, defined for $x \in[0,1]$, to the domain $(x, \xi) \in \mathcal{T}$ as follows:

$\tilde{\gamma}_{x}^{0}(x, \xi)=-\frac{1}{\lambda} \tilde{\gamma}^{0}(x, \xi) A+\frac{\sigma^{+-}(x)}{\lambda} \tilde{\gamma}^{1}(x, \xi)-L^{\alpha \alpha}(x, \xi) C$ $\tilde{\gamma}_{x}^{1}(x, \xi)=\frac{1}{\mu} \tilde{\gamma}^{1}(x, \xi) A-\frac{\sigma^{-+}(x)}{\mu} \tilde{\gamma}^{0}(x, \xi)+L^{\beta \alpha}(x, \xi) C$,

with boundary conditions

$$
\tilde{\gamma}^{0}(x, x)=\tilde{\gamma}^{1}(x, x)=0,
$$

and the relations

$$
\begin{aligned}
& \gamma_{0}(x)=\tilde{\gamma}^{0}(x, 0) \\
& \gamma_{1}(x)=\tilde{\gamma}^{1}(x, 0) .
\end{aligned}
$$

This set of PDEs, together with (11)-(14) can be solved using the procedure detailed in [11, Theorem 3.2]. Furthermore, since all coefficients are continuous, it can be shown that the unique solution obtained is in fact in $\mathcal{C}(\mathcal{T})$ componentwise (see [8]). This regularity of solution to the PDEs implies that the solution to the original ODEs is in $\left(\mathcal{C}^{1}([0,1])\right)^{p}$. This concludes the proof.

Applying the backstepping transformation defined in (8)-(10) to the original system (3)-(6) yields

$$
\begin{aligned}
& \alpha_{t}(t, x)+\lambda \alpha_{x}(t, x)=0 \\
& \beta_{t}(t, x)-\mu \beta_{x}(t, x)=0 \\
& \dot{X}(t)=A X(t)+B \beta(t, 0),
\end{aligned}
$$

with the following boundary conditions

$$
\begin{aligned}
\alpha(t, 0)= & q \beta(t, 0)+C X(t) \\
\beta(t, 1)= & \rho \alpha(t, 1)+U(t)+\left(\rho \gamma_{0}(1)-\gamma_{1}(1)\right) X(t) \\
& -\int_{0}^{1}\left(N^{\alpha}(\xi) \alpha(t, \xi)+N^{\beta}(\xi) \beta(t, \xi)\right) \mathrm{d} \xi,
\end{aligned}
$$

where

$$
\begin{aligned}
& N^{\alpha}(\xi)=L^{\beta \alpha}(1, \xi)-\rho L^{\alpha \alpha}(1, \xi) \\
& N^{\beta}(\xi)=L^{\beta \beta}(1, \xi)-\rho L^{\alpha \beta}(1, \xi) .
\end{aligned}
$$

The associated initial condition, denoted $\left(\alpha_{0}, \beta_{0}, X_{0}\right)$, is related to the initial condition $\left(u_{0}, v_{0}, X_{0}\right)$ by the inverse of the transformation (8)-(10). Differentiating (8)(9) with respect to time and space and using the boundary conditions (11)-(20), one can check that it maps the system (23)-(27) to the initial system (3)-(6). Due to the invertibility of the Volterra transformation (8)-(10), the two systems (23)-(27) and (3)-(6) are then equivalent. Thus, the stabilization of (23)-(27) implies the stabilization of the original system (3)-(6), and conversely.

For the control design of the target system (23)-(25) with the boundary control (26)-(27), we decompose the control input $U(t)$ as

$$
U(t)=U_{O D E}(t)+U_{B S}(t)
$$

where $U_{O D E}(\cdot)$ has to be designed for the stabilization of the ODE dynamics (25),

$$
\begin{aligned}
U_{B S}(t)= & -\kappa \alpha(t, 1)-\left(\rho \gamma_{0}(1)-\gamma_{1}(1)\right) X(t) \\
& +\int_{0}^{1}\left(N^{\alpha}(\xi) \alpha(t, \xi)+N^{\beta}(\xi) \beta(t, \xi)\right) \mathrm{d} \xi
\end{aligned}
$$


and the coefficient $\kappa$ is chosen such that

$$
|\kappa q|+|\rho q|<1
$$

The existence of such a $\kappa$ is a consequence of Assumption 2. The particular choice of a value of $\kappa$ verifying this inequality provides a tuning parameter for the control design. Note that if $\kappa$ does not satisfy (32), it is straightforward to adjust the proof of [1] and prove that the system is not robust to arbitrary small delays.

Remark that, due to the invertibility of the Volterra transformation (8)-(9), $U_{B S}(t)$ can be expressed in terms of $u, v$ and $X$.

The purpose of such a control law is to dissociate the stabilization of the ODE to the stabilization of the PDE. More precisely, the control law $U_{B S}(t)$ is designed to eliminate in-domain couplings. It preserves some proximal reflection in the target system (with the coefficient $\kappa$ ) to ensure delay-robustness [1]. This control, by itself, would guarantee the delay-robust exponential stabilization of (3)-(6) without the ODE subsystem (as shown in [1]), however, the presence of an ODE (even a stable one) may easily destabilize the coupled system.

In the next section, we will use (30) and (31) to rewrite (23)-(27) as a neutral delay differential equation with control input $U_{O D E}(t)$. It becomes then possible to derive a control law using classical methods [13-15] to ensure exponential stabilization.

\subsection{A neutral delay differential equation}

The equations (23)-(24) are transport equations, and consequently, for any $x \in[0,1]$, we get

$$
\begin{aligned}
& \alpha(t, x)=\alpha\left(t-\frac{x}{\lambda}, 0\right), t \geq \frac{x}{\lambda} \\
& \beta(t, x)=\beta\left(t-\frac{1-x}{\mu}, 1\right), t \geq \frac{1-x}{\mu} .
\end{aligned}
$$

The substitution of (30) and (31) in the boundary condition (27) and the use of (33) lead to,

$$
\beta(t, 1)=(\rho-\kappa) \alpha\left(t-\frac{1}{\lambda}, 0\right)+U_{O D E}(t) .
$$

Denoting $\tau=\frac{1}{\lambda}+\frac{1}{\mu}$, it follows from (26), (34) and (35) that, for any $t \geq \tau$,

$$
\begin{aligned}
\beta(t, 1)= & q(\rho-\kappa) \beta(t-\tau, 1)+(\rho-\kappa) C X\left(t-\frac{1}{\lambda}\right) \\
& +U_{O D E}(t) .
\end{aligned}
$$

For almost every $t<\tau, \beta(t, 1)$ remains bounded and can be expressed as a function of $\left(\alpha_{0}, \beta_{0}, X_{0}\right)$. Conse- quently (using the inverse of the backstepping transformation (8)-(9)) it can be expressed as a function of $\left(u_{0}, v_{0}, X_{0}\right)$, the initial condition of the PDE (3)-(5).

The ODE dynamics in (25) can be written as

$$
\dot{X}(t)=A X(t)+B \beta\left(t-\frac{1}{\mu}, 1\right) .
$$

This yields, for any $t \geq \tau+\frac{1}{\mu}$,

$$
\begin{aligned}
& \dot{X}(t)-(\rho-\kappa) q \dot{X}(t-\tau)=A X(t)-(\rho-\kappa) q A X(t-\tau) \\
& +B \beta\left(t-\frac{1}{\mu}, 1\right)-(\rho-\kappa) q B \beta\left(t-\frac{1}{\mu}-\tau, 1\right)
\end{aligned}
$$

Thus, using equation (36), we can substitute the term $\beta\left(t-\frac{1}{\mu}, 1\right)$ by an expression that only depends on $X$ and $U_{O D E}$, that is

$$
\begin{aligned}
& \dot{X}(t)-(\rho-\kappa) q \dot{X}(t-\tau)=A X(t)-(\rho-\kappa) q A X(t-\tau) \\
& +(\rho-\kappa) B C X(t-\tau)+B U_{O D E}\left(t-\frac{1}{\mu}\right)
\end{aligned}
$$

Note that this expression still holds for $\tau \leq t \leq \tau+\frac{1}{\mu}$. Taking the Laplace transform and denoting

$$
\hat{\phi}(s)=1-(\rho-\kappa) q \mathrm{e}^{-\tau s},
$$

one obtains

$$
(s I-A) \hat{\phi}(s) \hat{X}(s)=B \mathrm{e}^{-\frac{s}{\mu}} \hat{\tilde{U}}_{O D E}(s),
$$

where $\hat{\tilde{U}}_{O D E}(s)=\hat{U}_{O D E}(s)+(\rho-\kappa) C \mathrm{e}^{-\frac{s}{\lambda}} \hat{X}(s)$.

Under Assumption 2, the roots of the characteristic equation associated to (38) have right-bounded real parts. Thus, there exists a spectral exponential bound for the existence of the Laplace transform for (39)-(40).

\subsection{Spectral stabilization}

We are now able to design the control law $\hat{\tilde{U}}_{O D E}(s)$ that stabilizes (40). Denoting $\hat{Y}(s)=\hat{\phi}(s) \hat{X}(s)$, equation (40) can be rewritten as

$$
(s I-A) \hat{Y}(s)=B \mathrm{e}^{-\frac{s}{\mu}} \hat{\tilde{U}}_{O D E}(s) .
$$

Due to the detectability of $X$ from the new variable $Y$, we can reduce the stabilization problem of the neutral equation (40) into that of a finite-dimensional system with delayed input, that can be rewritten in time domain as

$$
\dot{Y}(t)=A Y(t)+B \tilde{U}_{O D E}\left(t-\frac{1}{\mu}\right), t \geq \frac{1}{\mu} .
$$


Different methods [28] can be used to design a control law that stabilizes equation (42). A classical result from [18] states that any control law that stabilizes such an equation is equivalent to a predictor. We then have the following Lemma.

Lemma 2 Take $A, B$ and $K$ verifying Assumption 1 and any $\kappa$ such that (32) holds. Then, the control law

$\tilde{U}_{O D E}(t)=K\left(\mathrm{e}^{\frac{A}{\mu}} Y(t)+\int_{t-\frac{1}{\mu}}^{t} \mathrm{e}^{A(t-\nu)} B \tilde{U}_{O D E}(\nu) \mathrm{d} \nu\right)$

exponentially stabilizes $Y(t)$ in (42). Furthermore, the state feedback

$$
U_{O D E}(t)=\tilde{U}_{O D E}(t)-(\rho-\kappa) C X\left(t-\frac{1}{\lambda}\right)
$$

exponentially stabilizes $X(t)$ in (38).

PROOF. For the state-predictor feedback $\tilde{U}_{O D E}(\cdot)$, the closed-loop system in (42) satisfies

$$
\dot{Y}(t)=(A+B K) Y(t), t \geq \frac{1}{\mu} .
$$

Exponential stability is guaranteed by the fact that $(A+$ $B K)$ is Hurwitz. By construction of $Y(t)$ and using (39), we have that $X(t)$, solution of (38), satisfies for any $t \geq$ $\tau$,

$$
X(t)=(\rho-\kappa) q X(t-\tau)+Y(t) .
$$

Since $|(\rho-\kappa) q|<1$ by $(32), X(t)$ is also exponentially stable.

We conclude this section with the following theorem.

Theorem 1 The control law

$$
U(t)=U_{O D E}(t)+U_{B S}(t),
$$

where $U_{B S}(t)$ is given in (31) and $U_{O D E}(t)$ is defined in Lemma 2, exponentially stabilizes in the sense of equation (7) the system (3)-(6) to its zero-equilibrium.

PROOF. We have proved in Lemma 2 that the control law $U(t)=U_{O D E}(t)+U_{B S}(t)$ exponentially stabilizes $X(t)$ and $Y(t)$ described by (38) and (42), respectively. Furthermore, according to the decomposition introduced in (40), the state-predictor feedback in Lemma 2 can be written as

$$
\tilde{U}_{O D E}(t)=K Y\left(t+\frac{1}{\mu}\right)
$$

which implies that $\tilde{U}_{O D E}(\cdot)$ exponentially converges to zero. Consequently, using $(32)$, the state $\beta(t, 1)$ governed by (36) exponentially converges to zero, which in turn implies from $(34)$ that $\beta(t, \cdot)$ converges $L^{2}$ exponentially to zero.

This implies, from (33) and the boundary condition (26), that $\alpha(t, \cdot)$ converges also $L^{2}$-exponentially to zero. This yields the existence of $\kappa_{0}>0$ such that $\|(\alpha, \beta, X)\| \leq \kappa_{0} \mathrm{e}^{-\nu t}\left\|\left(\alpha_{0}, \beta_{0}, X_{0}\right)\right\|$. Thus the control law $U(t)=U_{O D E}(t)+U_{B S}(t)$ ensures the exponential stabilization of (23)-(27). Due to the invertibility of the backstepping transformation (8)-(9), it is straightforward to prove the stabilization of (3)-(6).

Using a backstepping approach combined with a timedelay approach, we have derived a control law ensuring the exponential stabilization of (3)-(6) to its zero equilibrium. We need now to prove that this control law is delay-robust. This is the purpose of the next section.

\section{Delay-robust stabilization}

In this section we prove the delay-robustness of the control law designed in the previous section. Let us consider a small positive delay $\delta>0$ on the actuation input $U(\cdot)$. We now get from (27), (26), (33) and (34)

$$
\begin{aligned}
& \beta(t, 1)=\rho \alpha\left(t-\frac{1}{\lambda}, 0\right)+U(t-\delta)+\left(\rho \gamma_{0}(1)-\gamma_{1}(1)\right) X(t) \\
& -\int_{0}^{1}\left(N^{\alpha}(\xi) \alpha(t, \xi)+N^{\beta}(\xi) \beta(t, \xi)\right) \mathrm{d} \xi \\
& =\rho \alpha\left(t-\frac{1}{\lambda}, 0\right)+U(t-\delta)+\left(\rho \gamma_{0}(1)-\gamma_{1}(1)\right) X(t) \\
& \quad-\int_{0}^{1}\left(N^{\alpha}(\xi) \alpha\left(t-\frac{\xi}{\lambda}, 0\right)+N^{\beta}(\xi) \beta\left(t-\frac{1-\xi}{\mu}, 1\right)\right) \mathrm{d} \xi \\
& =q \rho \beta(t-\tau, 1)+U(t-\delta)+\rho C X\left(t-\frac{1}{\lambda}\right) \\
& \quad+\left(\rho \gamma_{0}(1)-\gamma_{1}(1)\right) X(t)-\int_{0}^{\tau} \tilde{N}(\xi) \beta(t-\xi, 1) \mathrm{d} \xi \\
& -\int_{0}^{1} N^{\alpha}(\xi) C X\left(t-\frac{\xi}{\lambda}\right) \mathrm{d} \xi
\end{aligned}
$$

where

$$
\tilde{N}(\xi)=\left\{\begin{array}{ll}
\mu N^{\beta}(1-\mu \xi) & \text { for } \quad \xi \in\left[0, \frac{1}{\mu}\right) \\
\lambda q N^{\alpha}\left(\lambda \xi-\frac{\lambda}{\mu}\right) & \text { for } \quad \xi \in\left(\frac{1}{\mu}, \tau\right]
\end{array} .\right.
$$

The function $\tilde{N}(\cdot)$ has therefore a unique extension to the whole interval $[0, \tau]$ that is $\mathcal{C}^{0}$ on $\left[0, \frac{1}{\mu}\right]$ and also a unique extension to that interval that is $\mathcal{C}^{0}$ on $\left[\frac{1}{\mu}, \tau\right]$ (depending only on the value assigned at $\left.\frac{1}{\mu}\right)$. These extensions are $k_{1}$-Lipschitz on $\left[0, \frac{1}{\mu}\right]$ and $k_{2}$-Lipschitz on $\left[\frac{1}{\mu}, \tau\right]$, respectively. However, there is in general a discontinuity 
at $\frac{1}{\mu}$ such that

$$
\tilde{N}\left(\frac{1}{\mu^{-}}\right)-\tilde{N}\left(\frac{1}{\mu^{+}}\right)=\left(\gamma_{1}(1)-\rho \gamma_{0}(1)\right) B .
$$

Since for integration purposes these two extensions are equivalent, and to avoid unnecessarily complex notation, depending on the context we may refer to one or the other as $\tilde{N}(\cdot)$.

Substituting the expression of $U(t)$ in (30) into (43) yields

$$
\begin{aligned}
& \beta(t, 1)=q \rho \beta(t-\tau, 1)-\kappa q \beta(t-\tau-\delta, 1)+U_{O D E}(t-\delta) \\
& +\rho C X\left(t-\frac{1}{\lambda}\right)-\kappa C X\left(t-\frac{1}{\lambda}-\delta\right) \\
& +\left(\rho \gamma_{0}(1)-\gamma_{1}(1)\right)(X(t)-X(t-\delta)) \\
& -\int_{0}^{\tau} \tilde{N}(\xi)(\beta(t-\xi, 1)-\beta(t-\xi-\delta, 1)) \mathrm{d} \xi \\
& -\int_{0}^{1} N^{\alpha}(\xi) C\left(X\left(t-\frac{\xi}{\lambda}\right)-X\left(t-\frac{\xi}{\lambda}-\delta\right)\right) \mathrm{d} \xi .
\end{aligned}
$$

Taking the Laplace transform of (44) and multiplying by $B$ one can get

$$
\begin{aligned}
& B \hat{\beta}(s, 1)-q \rho B \mathrm{e}^{-\tau s} \hat{\beta}(s, 1)+\kappa q \mathrm{e}^{-(\tau+\delta) s} B \hat{\beta}(s, 1) \\
+ & \int_{0}^{\tau} \tilde{N}(\xi)\left(\mathrm{e}^{-\xi s}-\mathrm{e}^{-(\xi+\delta) s}\right) \mathrm{d} \xi B \hat{\beta}(s, 1)=\mathrm{e}^{-\delta s} B \hat{U}_{O D E}(s) \\
& +B C\left(\rho \mathrm{e}^{-\frac{1}{\lambda} s}-\kappa \mathrm{e}^{-\left(\frac{1}{\lambda}+\delta\right) s}\right) \hat{X}(s) \\
& +B\left(\rho \gamma_{0}(1)-\gamma_{1}(1)\right)\left(1-\mathrm{e}^{-\delta s}\right) \hat{X}(s) \\
& -\int_{0}^{1} N^{\alpha}(\xi) B C\left(\mathrm{e}^{-\frac{\xi}{\lambda} s}-\mathrm{e}^{-\left(\frac{\xi}{\lambda}+\delta\right) s}\right) \mathrm{d} \xi \hat{X}(s) .
\end{aligned}
$$

The Laplace transform of equation (37) implies that $(s I-A) \hat{X}(s)=B \mathrm{e}^{-\frac{s}{\mu}} \hat{\beta}(s, 1)$. Moreover, using the expression of the state feedback in Lemma 2, we have

$$
\begin{aligned}
\hat{U}_{O D E}(s) & =\hat{\tilde{U}}_{O D E}(s)-(\rho-\kappa) C \mathrm{e}^{-\frac{s}{\lambda}} \hat{X}(s) \\
& =K_{0}(s) \hat{\phi}(s) \hat{X}(s)-(\rho-\kappa) C \mathrm{e}^{-\frac{s}{\lambda}} \hat{X}(s),
\end{aligned}
$$

where $K_{0}(s)$ stands for the Laplace transform of the predictor state feedback in Lemma 2, namely

$$
K_{0}(s)=\left[I-K(s I-A)^{-1}\left(I-\mathrm{e}^{-(s I-A) \frac{1}{\mu}}\right) B\right]^{-1} K \mathrm{e}^{\frac{A}{\mu}} .
$$

In what follows, we denote

$$
\begin{aligned}
\hat{\phi}_{1}(s, \delta)= & 1-q \rho \mathrm{e}^{-\tau s}+\kappa q \mathrm{e}^{-(\tau+\delta) s} \\
& +\left(1-\mathrm{e}^{-\delta s}\right) \int_{0}^{\tau} \tilde{N}(\xi) \mathrm{e}^{-\xi s} \mathrm{~d} \xi
\end{aligned}
$$

Multiplying equation (45) by $\mathrm{e}^{-\frac{s}{\mu}}$ and using (46), we obtain

$$
\begin{aligned}
& (s I-A)\left(\hat{\phi}_{1}(s, \delta)\right) \hat{X}(s)=B \mathrm{e}^{-\frac{s}{\mu}}\left[C \mathrm{e}^{-\frac{s}{\lambda}}\left(\rho-\kappa \mathrm{e}^{-\delta s}\right)\right. \\
& +\mathrm{e}^{-\delta s} K_{0}(s) \hat{\phi}(s)-(\rho-\kappa) C \mathrm{e}^{-\frac{s}{\lambda}-s \delta} \\
& +\left(\rho \gamma_{0}(1)-\gamma_{1}(1)\right)\left(1-\mathrm{e}^{-\delta s}\right) \\
& \left.-\left(1-\mathrm{e}^{-\delta s}\right) \int_{0}^{1} N^{\alpha}(\xi) C \mathrm{e}^{-\frac{\xi s}{\lambda}} \mathrm{d} \xi\right] \hat{X}(s)
\end{aligned}
$$

where $\hat{\phi}$ is defined in (39).

From $\left[26\right.$, Theorem 1], we know that $\phi_{1}(\cdot, \delta) \in \mathcal{A}$ has a unique inverse in $\mathcal{A}$ if and only if

$$
\inf _{\operatorname{Re}(s) \geq 0}\left|\hat{\phi}_{1}(s, \delta)\right|>0 .
$$

We have the following lemma on invertibility of $\hat{\phi}_{1}(s, \delta)$ in $\hat{\mathcal{A}}$ (where the Banach algebra $\hat{\mathcal{A}}$ is defined in section 2.1).

Lemma 3 There exists $\delta^{\star} \in(0, \tau]$ such that

$$
\inf _{\delta \in\left[0, \delta^{\star}\right]} \inf _{\operatorname{Re}(s) \geq 0}\left|\hat{\phi}_{1}(s, \delta)\right|>0
$$

PROOF. Consider a fixed $\delta \in\left[0, \min \left(\frac{1}{\mu}, \frac{1}{\lambda}\right)\right]$. The element $\hat{\phi}_{1}(s, \delta)$ lies in $\hat{\mathcal{A}}$, since $\tilde{N}(\cdot)$ is in $L^{1}\left(\mathbb{R}^{+}, \mathbb{R}\right)$. Furthermore, we have that $\hat{\phi}_{1}(s, \delta)$ is invertible in the Banach algebra $\hat{\mathcal{A}}$ provided that $\left\|1-\hat{\phi}_{1}(s, \delta)\right\|_{\hat{\mathcal{A}}}<1$. Since $\tilde{N}(\cdot)$ with support in $[0, \tau]$ belongs to $L^{\infty}([0, \tau], \mathbb{R})$, a direct calculation using the triangular inequality for the $L^{1}$-norm shows that

$$
\begin{aligned}
& \left\|1-\hat{\phi}_{1}(s, \delta)\right\|_{\hat{\mathcal{A}}} \leq|q \rho|+|\kappa q|+\int_{0}^{\delta}|\tilde{N}(\xi)| \mathrm{d} \xi \\
& +\int_{\delta}^{\frac{1}{\mu}}|\tilde{N}(\xi)-\tilde{N}(\xi-\delta)| \mathrm{d} \xi+\int_{\frac{1}{\mu}+\delta}^{\tau}|\tilde{N}(\xi)-\tilde{N}(\xi-\delta)| \mathrm{d} \xi \\
& +\int_{\frac{1}{\mu}}^{\frac{1}{\mu}+\delta}(|\tilde{N}(\xi-\delta)|+|\tilde{N}(\xi)|) \mathrm{d} \xi+\int_{\tau}^{\tau+\delta}|\tilde{N}(\xi-\delta)| \mathrm{d} \xi
\end{aligned}
$$

Since $\tilde{N}(\cdot)$ is $k_{1}$-Lipschitz in $\left[0, \frac{1}{\mu}\right]$ and $k_{2}$-Lipschitz in $\left[\frac{1}{\mu}, \tau\right]$, we get

$$
\left\|1-\hat{\phi}_{1}(s, \delta)\right\|_{\hat{\mathcal{A}}} \leq|q \rho|+|\kappa q|+\delta\left(4\|\tilde{N}\|_{L^{\infty}}+\frac{k_{1}}{\mu}+\frac{k_{2}}{\lambda}\right) .
$$

Noting that with the condition (32) we have $|q \rho|+|\kappa q|<$ 1 , there exists $\delta^{\star}>0$ with

$$
\delta^{\star}<\min \left(\frac{1-|q \rho|-|\kappa q|}{4\|\tilde{N}\|_{L^{\infty}}+\frac{k_{1}}{\mu}+\frac{k_{2}}{\lambda}}, \min \left(\frac{1}{\mu}, \frac{1}{\lambda}\right)\right)
$$


such that for any $\delta \in\left[0, \delta^{\star}\right],\left\|1-\hat{\phi}_{1}(s, \delta)\right\|_{\hat{\mathcal{A}}}<1$. This implies that $\phi_{1}(t, \delta)$ is a unit of $\mathcal{A}$, that is (49) holds. $\square$

One can now fully understand the importance of the choice of $\kappa$ made in (32). This choice is possible due to Assumption 2.

Equation (48) yields

$$
\begin{aligned}
& (s I-A)\left(\hat{\phi}_{1}(s, \delta)\right) \hat{X}(s)=B \mathrm{e}^{-\frac{s}{\mu}}\left[C \mathrm{e}^{-\frac{s}{\lambda}}\left(\rho-\kappa \mathrm{e}^{-\delta s}\right)\right. \\
& -\left(1-\mathrm{e}^{-\delta s}\right) \int_{0}^{1} N^{\alpha}(\xi) C \mathrm{e}^{-\frac{\xi s}{\lambda}} \mathrm{d} \xi-(\rho-\kappa) C \mathrm{e}^{-\frac{s}{\lambda}-s \delta} \\
& +\left(\rho \gamma_{0}(1)-\gamma_{1}(1)\right)\left(1-\mathrm{e}^{-\delta s}\right)+\mathrm{e}^{-\delta s} K_{0}(s) \hat{\phi}(s) \\
& \left.-K_{0}(s) \hat{\phi}_{1}(s, \delta)+K_{0}(s) \hat{\phi}_{1}(s, \delta)\right] \hat{X}(s) .
\end{aligned}
$$

We consequently get the following characteristic quasipolynomial $p(s)$

$$
\begin{aligned}
& \operatorname{det}\left(\left(s I-A-B K_{0}(s) \mathrm{e}^{-\frac{s}{\mu}}\right) \hat{\phi}_{1}(s, \delta)-B \mathrm{e}^{-\frac{s}{\mu}}\left(\rho C \mathrm{e}^{-\frac{1}{\lambda} s}\right.\right. \\
& -\kappa C \mathrm{e}^{-\frac{1}{\lambda} s-\delta s}-\left(1-\mathrm{e}^{-\delta s}\right) \int_{0}^{1} N^{\alpha}(\xi) C \mathrm{e}^{-\frac{\xi}{\lambda} s} \mathrm{~d} \xi \\
& +\mathrm{e}^{-\delta s} K_{0}(s) \hat{\phi}(s)-(\rho-\kappa) C \mathrm{e}^{-\frac{s}{\lambda}-s \delta}-K_{0}(s) \hat{\phi}_{1}(s, \delta) \\
& \left.+\left(\rho \gamma_{0}(1)-\gamma_{1}(1)\right)\left(1-\mathrm{e}^{-\delta s}\right)\right)=0
\end{aligned}
$$

Let us now denote

$$
\begin{gathered}
F(s)=\left(s I-\left(A+B K_{0}(s) \mathrm{e}^{-\frac{s}{\mu}}\right)\right) \hat{\phi}_{1}(s, \delta) \\
H(s)=B \mathrm{e}^{-\frac{s}{\mu}}\left(\rho \gamma_{0}(1)-\gamma_{1}(1)+\rho C \mathrm{e}^{-\frac{s}{\lambda}}+\left(\rho q \mathrm{e}^{-\tau s}-1\right.\right. \\
\left.\left.-\int_{0}^{\tau} \tilde{N}(\xi) \mathrm{e}^{-\xi s} \mathrm{~d} \xi\right) K_{0}(s)-\int_{0}^{1} N^{\alpha}(\xi) C \mathrm{e}^{-\frac{\xi}{\lambda} s} \mathrm{~d} \xi\right) .
\end{gathered}
$$

Using the definitions of $\hat{\phi}(s)$ and $\hat{\phi}_{1}(s, \delta)$, equation (50) can be rewritten as

$$
p(s)=\operatorname{det}\left(F(s)-\left(1-\mathrm{e}^{-\delta s}\right) H(s)\right)=0 .
$$

Since $K_{0}(s)$ is bounded in the right-half plane, $H(s)$ is bounded in the right-half plane. We are now finally able to prove that the control law $U(t)$ as defined in (30) delay-robustly stabilizes the system (3)-(6).

Theorem 2 The control law $U(t)=U_{O D E}(t)+U_{B S}(t)$ as defined in (30) delay-robustly stabilizes the system (3)(6). That is, there exists $\delta^{\star}>0$ such that, for all $\delta \in$ $\left[0, \delta^{\star}\right], U(t)=U_{O D E}(t-\delta)+U_{B S}(t-\delta)$ exponentially stabilizes the system $(3)-(6)$.

PROOF. The closed-loop characteristic equation can be written as in (53), where $F(s)$ has all its roots in the left-half complex plane (see Lemma 3 ), and $H(s)$ is bounded in the right-half complex plane. By contradiction, assume that there exists $z \in \mathbb{C}, z \neq 0$ and $\operatorname{Re}(z) \geq$
0 , such that $p(z)=0$. There exists $\eta \neq 0$ such that

$$
F(z) \eta=\left(1-\mathrm{e}^{-\delta z}\right) H(z) \eta
$$

This yields

$$
\eta^{*} F^{*}(z) F(z) \eta=\left|1-\mathrm{e}^{-\delta z}\right|^{2} \eta^{*} H^{*}(z) H(z) \eta,
$$

where $^{*}$ denotes the conjugate transpose. Since $F(z)$ is non singular in $\mathbb{C}^{+}$, there exists $M_{0}>0$ such that $M_{0}<$ $\eta^{*} F^{*}(z) F(z) \eta$. Similarly, $H(z)$ is bounded in $\mathbb{C}^{+}$, so that there exists $M_{1}>0$ such that

$$
M_{0} \leq\left|1-\mathrm{e}^{-\delta z}\right|^{2} \eta^{*} H^{*}(z) H(z) \eta \leq\left|1-\mathrm{e}^{-\delta z}\right|^{2} M_{1} .
$$

Construct $\delta_{m}(z)=\frac{\bar{\delta}}{|z|}$, for some $\bar{\delta}>0$ such that $\mathrm{e}^{\bar{\delta}}<$ $1+\sqrt{\frac{M_{0}}{M_{1}}}$. It follows that for any $\delta \leq \delta_{m}(z)$,

$$
\left|1-\mathrm{e}^{-\delta z}\right| \leq \mathrm{e}^{\bar{\delta}}-1<\sqrt{\frac{M_{0}}{M_{1}}} .
$$

Since $p(s)$ has only a finite number of zeros in the righthalf plane, where the zeros have finite module [15], the quantity $\delta^{\star}=\min _{z} \delta_{m}(z)$ is strictly positive. This implies that for any $\delta \leq \delta^{\star}$, (54) holds. This leads to a contradiction with the previous inequality. Hence there does not exist any $z \in \mathbb{C}^{+}$such that $p(z)=0$. Furthermore, since the principal term of $p(s)$ is precisely the principal term of $\hat{\phi}_{1}(s, \delta)$ which is stable by construction (see Lemma 3 ), the asymptotic vertical chain of zeros of $p(s)$ can not be the imaginary axis. This implies delayrobust stability since all zeros of $p(s)$ are in the open left-half complex plane.

\section{Simulation results}

In this section we illustrate our results with simulations.

Let us consider the unstable system (3)-(6) for which the coefficients are defined by

$$
\begin{aligned}
& \lambda=\mu=\sigma^{+-}=\sigma^{-+}=q=1, \quad \rho=0.6 . \\
& A=0.1, B=0.1, C=0.2 .
\end{aligned}
$$

The parameters values are chosen such that

- the ODE and the PDE open-loop system are unstable [3],

- the reflexion terms satisfy $0<|\rho q|<1$, so that Assumption 2 is fulfilled.

We consider the norm $\|\cdot\|$ defined by (1). The initial condition is chosen as a $C^{1}$ function. Similarly to [1], the condition (32) means that one cannot completely cancel the proximal reflexion term $\rho u(t, 1)$ to design a delayrobust control law when $|\rho q|>\frac{1}{2}$. To emphasize this 
property, we choose $|\rho q|=0.6>\frac{1}{2}$ in our simulations. The algorithm we use is adapted from the one proposed in [2]. Using the method of characteristics, we write the integral equations associated to the PDE-system (11)(20). These integral equations are solved using a fixedpoint algorithm. These kernels are then used to compute the control law. Finally, the original system (3)-(6) is simulated using a Godunov's discretization scheme. The predictor is adjusted from the one presented in [19]

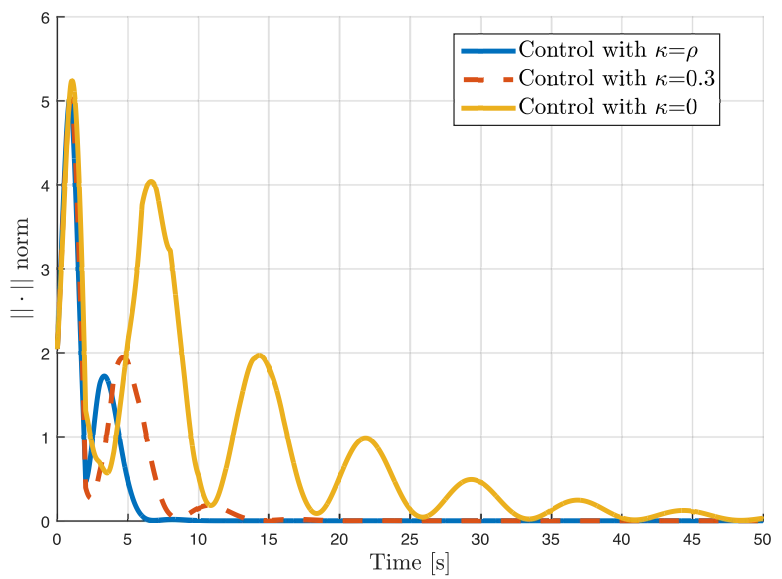

Fig. 2. Time evolution of the $\|\cdot\|$-norm of system (3)-(5) for the parameters (55)-(56) for different values of $\kappa$ without any delay

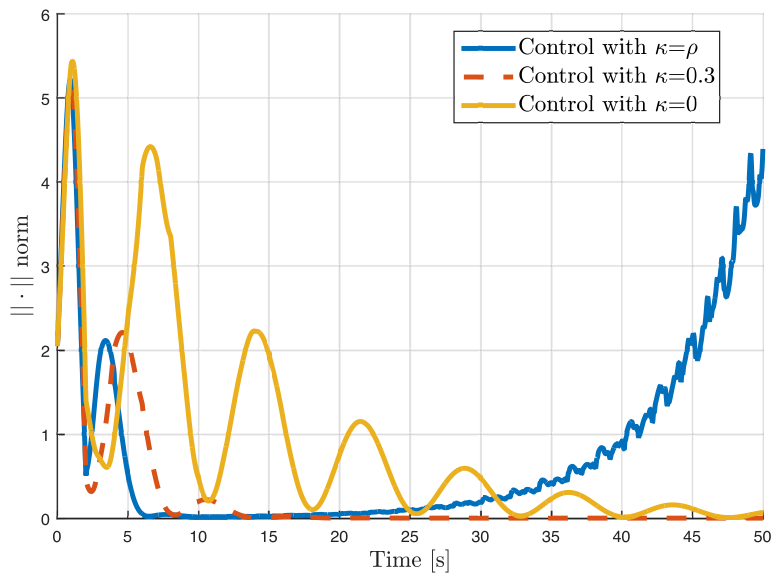

Fig. 3. Time evolution of the $\|\cdot\|$-norm of system (3)-(5) for the parameters (55)-(56) for different values of $\kappa$ in presence of a 0.02 s delay.

Figure 2 pictures the $\|\cdot\|$-norm of the state $(u, v, X)$ using the control law (31) for different values of $\kappa$ without any delay whereas a small delay in the loop $(\delta=0.02 \mathrm{~s})$ is considered in Figure 3. Choosing $\kappa$ so that (32) holds, the resulting stabilizing control law is delay-robust. For such a value of $\kappa$, due to the definition of $\|\cdot\|$, the state $X$ converges to zero. Figure 4 shows the evolution of $u(t, x)$ in presence of the delay $\delta=0.02 \mathrm{~s}$ for a value of $\kappa=0.3$. Note that the convergence is only guaranteed in the sense of (7). Finally, Figure 5 depicts the control effort for different values of $\kappa$ in presence of the delay $\delta=0.02 \mathrm{~s}$.

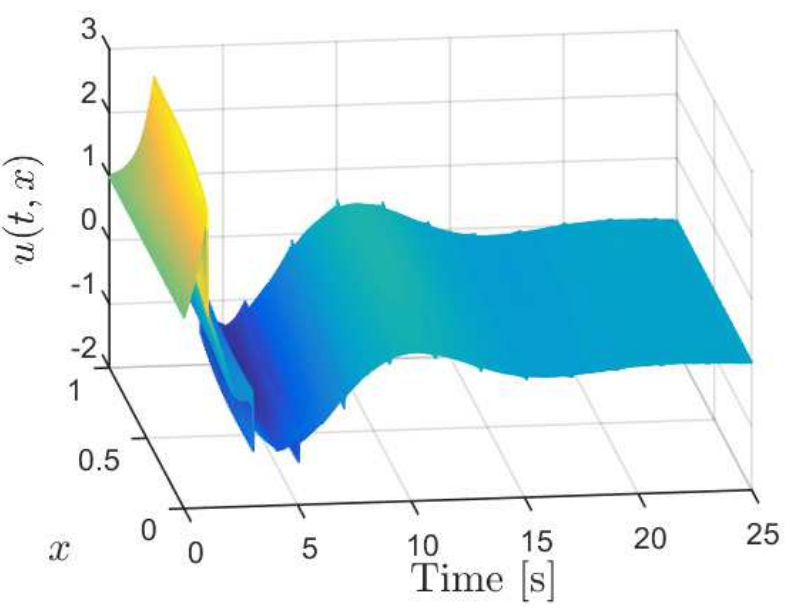

Fig. 4. Evolution of $u(t, x)$ for a value of $\kappa=0.3$ in presence of a 0.02 s delay.

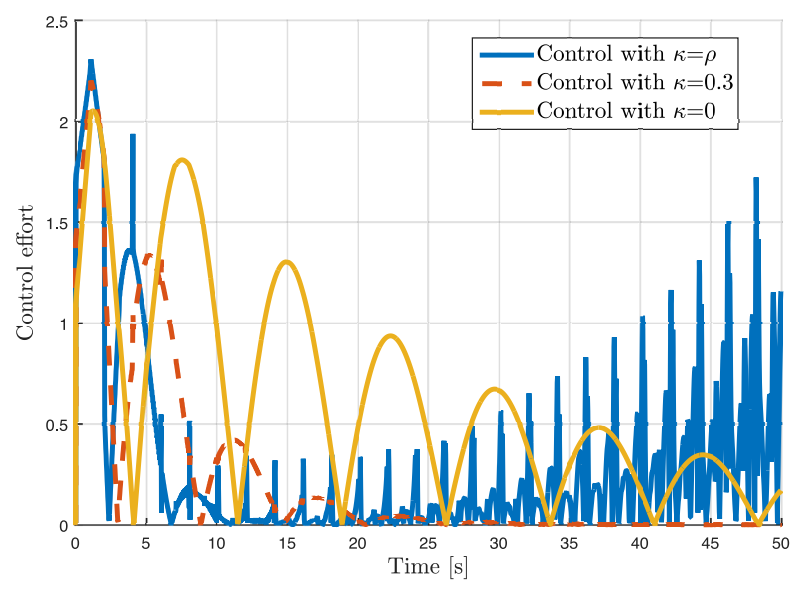

Fig. 5. Time evolution of the control effort $U(t)$ for different values of $\kappa$ in presence of a $0.02 \mathrm{~s}$ delay.

\section{Concluding remarks}

In this paper, a delay-robust stabilizing feedback control law was developed for a coupled hyperbolic PDE-ODE system. The proposed method combines a first feedback constructed using the backstepping approach with a second predictor-type feedback. The second feedback control is obtained after a suitable change of variables that reduces the stabilization problem of the PDE-ODE system to that of an ODE with input delay for which classical results for delay equations can be used. The robustness to small delays (in the actuation) of our combined feedback strategy is ensured by preserving some proximal reflection terms in the PDEs in the backstepping design. The degree to which these reflection terms are canceled introduces a tuning parameter that enables some trade-offs between convergence rates in the nominal system and delay-robustness. In future works, the 
delay-robustness properties of the output-feedback controller (crucial for application on an industrial problem) remains to be considered.

\section{References}

[1] J. Auriol, U.J.F. Aarsnes, P. Martin, and F. Di Meglio. Delayrobust control design for two heterodirectional linear coupled. Accepted in IEEE-Transactions on Automatic Control, 2017.

[2] J. Auriol and F. . Di Meglio. Minimum time control of heterodirectional linear coupled hyperbolic pdes. Automatica, 71:300-307, 2016.

[3] G. Bastin and J.-M. Coron. Stability and boundary stabilization of 1-D hyperbolic systems. Springer, 2016.

[4] N. Bekiaris-Liberis and M. Krstic. Nonlinear control under nonconstant delays. SIAM, 2013.

[5] N. Bekiaris-Liberis and M. Krstic. Compensation of wave actuator dynamics for nonlinear systems. IEEE Transactions on Automatic Control, 59(6):1555-1570, June 2014.

[6] D. Bresch-Pietri. Commande robuste de systèmes à retard variable: Contributions théoriques et applications au contrôle moteur. PhD thesis, Ecole Nationale Supérieure des Mines de Paris, 2012.

[7] D. Bresch-Pietri and M. Krstic. Adaptive output feedback for oil drilling stick-slip instability modeled by wave pde with anti-damped dynamic boundary. In American Control Conference (ACC), 2014, pages 386-391. IEEE, 2014.

[8] J.-M. Coron, R. Vazquez, M. Krstic, and G. Bastin. Local exponential $h^{2}$ stabilization of a $2 \times 2$ quasilinear hyperbolic system using backstepping. SIAM Journal on Control and Optimization, 51(3):2005-2035, 2013.

[9] S. Damak, M. Di Loreto, and S. Mondié. Stability of linear continuous-time difference equations with distributed delay: Constructive exponential estimates. International Journal of Robust and Nonlinear Control, 25(17):3195-3209, 2015.

[10] R. Datko, J. Lagnese, and M.P. Polis. An example on the effect of time delays in boundary feedback stabilization of wave equations. SIAM Journal on Control and Optimization, 24(1):152-156, 1986.

[11] Florent Di Meglio, Federico Bribiesca Argomedo, Long $\mathrm{Hu}$, and Miroslav Krstic. Stabilization of coupled linear heterodirectional hyperbolic pde-ode systems. Automatica, $87: 281-289,2018$.

[12] E. Fridman and U. Shaked. H $\infty$-control of linear state-delay descriptor systems: an LMI approach. Linear Algebra and its Applications, 351:271-302, 2002.

[13] K. Gu, V.L. Kharitonov, and J. Chen. Stability of time-delay systems. Birkhäuser, 2003.

[14] J.K. Hale and S.M. Verduyn Lunel. Introduction to functional differential equations. Springer-Verlag, 1993.

[15] J.K. Hale and S.M. Verduyn Lunel. Strong stabilization of neutral functional differential equations. IMA Journal of Mathematical Control and Information, 19(1 and 2):5-23, 2002.

[16] M. Krstic and A. Smyshlyaev. Backstepping boundary control for first-order hyperbolic PDEs and application to systems with actuator and sensor delays. Systems \& Control Letters, 57(9):750-758, 2008.

[17] H. Logemann, R. Rebarber, and G. Weiss. Conditions for robustness and nonrobustness of the stability of feedback systems with respect to small delays in the feedback loop. SIAM Journal on Control and Optimization, 34(2):572-600, 1996.

[18] L. Mirkin and N. Raskin. Every stabilizing deadtime controller has an observer-predictor-based structure. Automatica, 39:1747-1754, 2003.
[19] S. Mondié and W. Michiels. Finite spectrum assignment of unstable time-delay systems with a safe implementation. IEEE Transactions on Automatic Control, 48(12):2207-2212, 2003.

[20] S.-I. Niculescu. Delay effects on stability: a robust control approach, volume 269. Springer Science \& Business Media, 2001.

[21] S.-I. Niculescu. On delay-dependent stability under model transformations of some neutral linear systems. International Journal of Control, 74(6):609-617, 2001.

[22] C. Sagert, F. Di Meglio, M. Krstic, and P. Rouchon. Backstepping and flatness approaches for stabilization of the stick-slip phenomenon for drilling. IFAC Proceedings Volumes, 46(2):779-784, 2013.

[23] B. Saldivar, S. Mondié, S.-I. Niculescu, H. Mounier, and I. Boussaada. A control oriented guided tour in oilwell drilling vibration modeling. Annual Reviews in Control, 42:100 113, 2016.

[24] R. Sipahi, S.-I. Niculescu, C.T. Abdallah, W. Michiels, and K. Gu. Stability and stabilization of systems with time delay. IEEE Control Systems, 31(1):38-65, 2011.

[25] O. Smith. A controller to overcome dead time. ISA J., 6:2833, 1959.

[26] M. Vidyasagar. Input-output stability of a broad class of linear time-invariant multivariable systems. SIAM J. Control, 10(1):203-209, 1972.

[27] D. Yue and Q.-L. Han. Delayed feedback control of uncertain systems with time-varying input delay. Automatica, 41(2):233-240, 2005.

[28] Q.-C. Zhong. Robust control of time-delay systems. SpringerVerlag, 2006. 